\title{
Development of CAD/CAM Based Brace Models for the Treatment of Patients with Scoliosis-Classification Based Approach versus Finite Element Modelling
}

\author{
Hans-Rudolf Weiss ${ }^{1}$, Alexander Kleban ${ }^{2}$ \\ ${ }^{1}$ Orthopaedic Rehabilitation Services, Gesundheitsforum Nahetal, Gensingen, Germany \\ ${ }^{2}$ Department of Mathematics, Lomonosov Moscow State University, Moscow, Russia
}

Study Design: Retrospective controlled cohort study comparing the in-brace correction of two samples of scoliosis patients with braces of different computer aided design (CAD).

Purpose: In-brace correction and compliance correlate with outcome. The more standardized CAD braces that are available should enable improved in-brace correction and outcome. This study compared recent CAD brace developments with respect to in-brace corrections.

Overview of Literature: A 2013 randomized controlled trial demonstrated that 72\% of a population complying to Scoliosis Research Society inclusion criteria on bracing did not progress using braces (mainly Boston braces) used in the United States and Canada with moderate corrective effect.

Methods: In-brace corrections achieved in a sample of patients fulfilling the inclusion criteria for studies on bracing using the classification based approach (CBA) were compared to the recent individual CAD/computer aided manufacturing bracing based on finite element modelling approach (FEMA).

Results: In-brace corrections using the different approaches differed widely. CBA in-brace corrections were $66 \%$ of the initial value. FEMA in-brace correction was $42 \%$ of the initial value.

Conclusions: Considering the fact that in-brace correction (and compliance) determines the end result of bracing in the treatment of scoliosis, scoliosis braces based on CBA are superior to the FEMA and the standard plaster based brace applications.

Keywords: Scoliosis; Brace; Orthothis; Correction; Computer aided design

\section{Introduction}

Scoliosis is a lateral deviation of the spine commonly exhibiting different patterns of curvature [1]. The basic curve patterns are named after the location of the major curve (e.g., thoracic, lumbar, thoracolumbar, double major, and double thoracic), but other specific classifications have been described [2]. In structural scoliosis, there is usually a certain amount of spinal torsion and a disturbance of the sagittal profile coupled to the lateral defor-

Received Jan 29, 2015; Revised Mar 25, 2015; Accepted Mar 25, 2015

Corresponding author: Hans-Rudolf Weiss

Orthopaedic Rehabilitation Services, Gesundheitsforum Nahetal, Alzeyer Str. 23, Gensingen D-55457, Germany

Tel: +49-6727-894040, Fax: +49-6727-8940429, E-mail: hr.weiss@skoliose-dr-weiss.com 
mation [1]. Therefore, scoliosis must be more accurately regarded as a three-dimensional (3D) deformity of the spine and trunk, which may progress quickly during periods of rapid growth [1].

There is some evidence for the use of physiotherapy in the treatment of patients with spinal deformities. However, during the pubertal growth spurt (high-risk phase for progression), brace treatment is the most important mode of treatment [1]. In-brace correction and compliance correlate with outcome [1,3]. More asymmetric braces with increased corrective effect are preferable (Cheneau style) to the more symmetric Boston braces [1,3].

Historically, the Boston brace treatment has produced brace corrections exceeding 30\% of the initial angle of curvature. For Cheneau braces, as early as 1985 brace corrections exceeding an average of $40 \%$ have yielded beneficial end-results. Recent in-brace corrections with refined Cheneau derivates have reportedly exceeded $50 \%$ of the initial value. While the Cheneau brace has historically been constructed only by modifying the plaster positive of the individual patient (Fig. 1), the Boston brace was constructed by modification of a plaster cast individually or by adjustment of a formerly provided prefabricated Boston module.

The Boston brace is now regarded as the baseline standard for new individual computer aided design/computer aided manufacturing (CAD/CAM) braces [4-9]. Finite element modelling results have suggested that individual braces can be constructed using CAD to correct individual curvature patterns when low-dose stereo X-rays are available for calculation. This approach seems very promising, as it addresses very precisely curve patterns individually and not by classification. However, in-brace corrections still do not reach the percentage correction attained with Cheneau applications $[6,9]$.

Cheneau applications can also be constructed by CAD/ CAM $[1,2]$. These applications are not based on finite element modelling, but instead on specific classifications of curve patterns developed empirically (Fig. 2) [2]. The original Cheneau brace was derived from the Abbott model of correction [10]. Voids are implemented opposite to the pressure zones in all $3 \mathrm{D}$ to allow the $3 \mathrm{D}$ corrective movement without compression of the patient. This has lead to semi-standardized, custom-made braces for functional 3- and 4-curve patterns [10]. This simple Cheneau classification is based on the version implemented about a half-century ago [10]. This simple classification remains relevant for present-day physiotherapy treatment of scoliosis. However, more specific bracing classifications have been established.

The most recent classification for bracing scoliosis, the augmented Lehnert-Schroth classification (Fig. 2), derived from the original Lehnert-Schroth classification. The present publication provides a description of the empirically based CAD/CAM brace model (classification based approach, $\mathrm{CBA}$ ), which incorporates the collective information obtained over nearly 4 decades preceeding the actual CAD/CAM Cheneau derivates [2].

An ongoing prospective controlled trial is comparing this approach to other samples described in literature using the Scoliosis Research Society (SRS) inclusion criteria for studies on bracing. A recent publication described in-
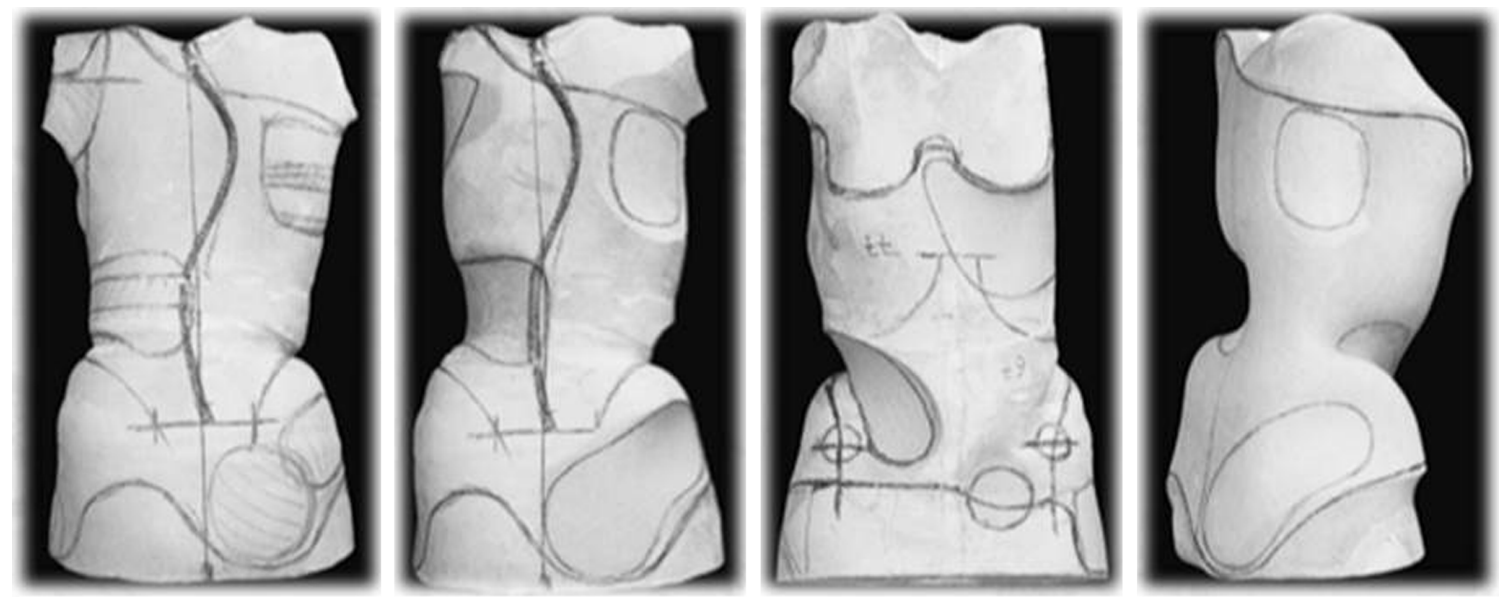

Fig. 1. Plaster based brace construction according to the Cheneau principles. 

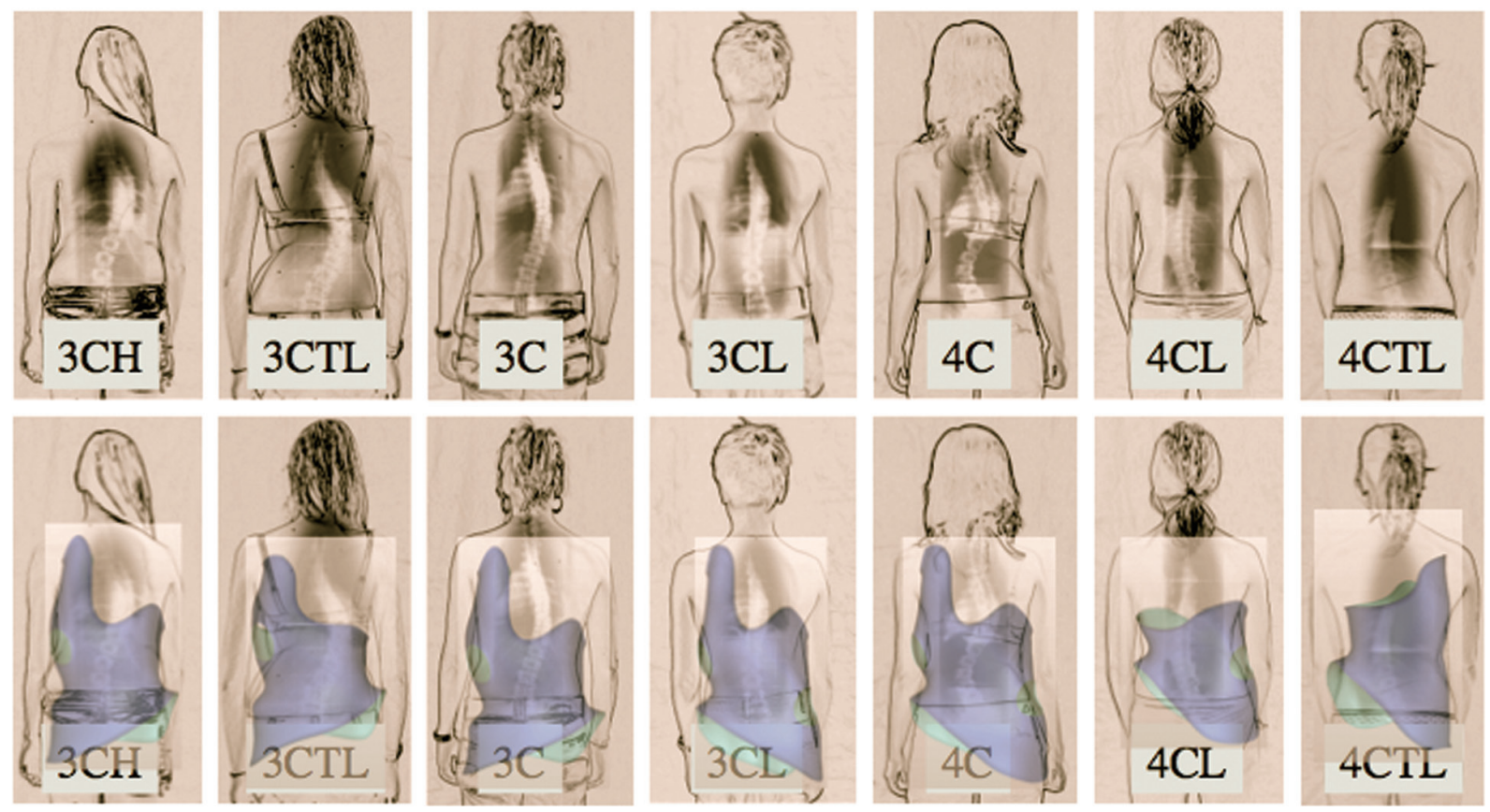

Fig. 2. The Augmented Lehnert-Schroth classification used for the selection of the appropriate brace from Gensingen library. 3CH, 3-curve with hip prominence; 3CTL, 3-curve with hip prominence thoracolumbar; 3C, 3-curve balanced; 3CL, 3-curve with long lumbar countercurve; 4C, 4-curve double; 4CL, 4-curve single lumbar; 4CTL, 4-curve single thoracolumbar. Reprinted from Weiss et al. [2], according to the Creative Commons License of OA Publishing London.

brace corrections achieved using the finite element modelling approach (FEMA) [11]. So, the in-brace corrections achieved in the sub-sample of patients fulfilling SRS inclusion criteria for studies on bracing using the CBA [2] can be compared to the individual CAD/CAM bracing approach based on FEMA.

\section{Materials and Methods}

Within the CBA, seven basic patterns of curvature (Figs. 2-4) were established as a subclassification to the original Lehnert-Schroth classification (3- and 4-curve). Two additional patterns were introduced to address double thoracic curvatures and a thoracolumbar pattern with a structural high thoracic counter curve. In 2011, we commenced a prospective controlled study on CBA outcome using the SRS inclusion criteria involving all patients available at that time, with the aim of testing the in-brace correction achieved. Twenty-one patients fulfilled the SRS inclusion criteria on bracing. The average Cobb curvature angle and in-brace Cobb angle was $31^{\circ}$ and $11^{\circ}$, respectively. Concerning FEMA [11], until recently there were no consistent results regarding the published inbrace correction [9]. The in-brace corrections achieved

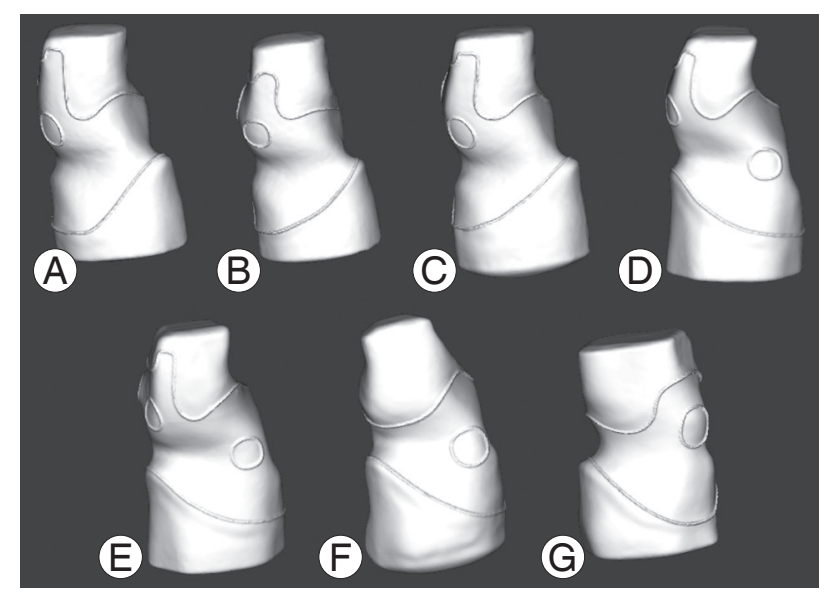

Fig. 3. The seven basic models of the Gensingen library. Model (A) 3BH, (B) 3BTL, (C) 3BN, (D) 3BL, (E) 4B, (F) 4BL, (G) 4BTL. 3B, functional 3 curve pattern; $4 B$, functional 4 curve pattern. Reprinted from Weiss [12], according to the Creative Commons License of OA Publishing London.

using FEMA could derived from a sample of 15 patients reported on recently [11]. We statistically compared the average in-brace corrections as achieved with the two different approaches. The Student $t$-test was used to compare the in-brace corrections of the two different samples. 


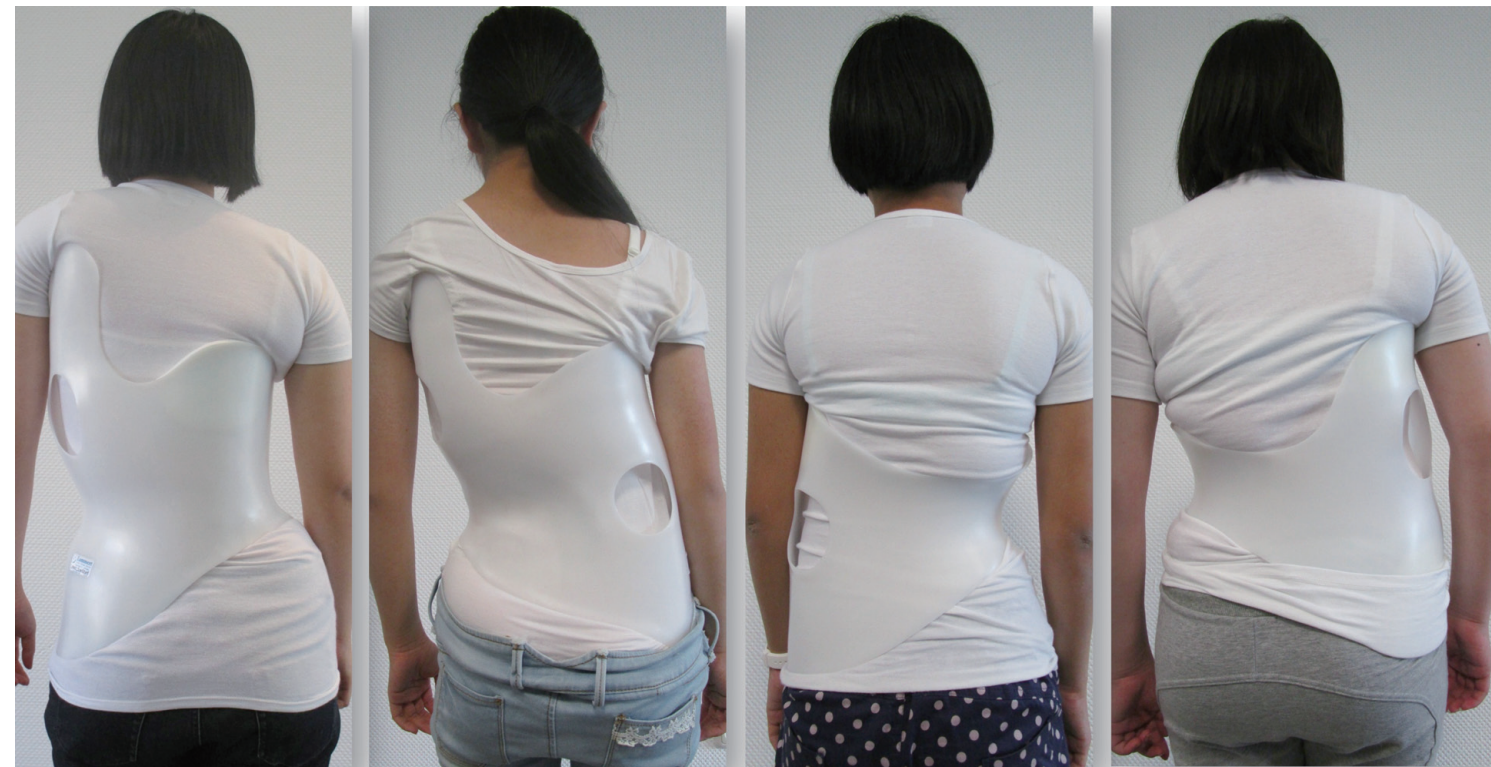

Fig. 4. Different models from the Gensingen brace computer aided design/computer aided manufacturing (CAD/CAM) library

\section{Results}

CBA analyses involved 21 females who matched the SRS inclusion criteria for studies on bracing. Their average age was 12.2 years (standard deviation [SD], 1.1 years). The distribution of curve patterns was thoracic $(\mathrm{n}=11)$, double major $(n=4)$, lumbar $(n=4)$, and thoracolumbar $(n=2)$. The average Risser stage was 0.38 (SD, 0.68) and the average Cobb angle was $31.33^{\circ}\left(\mathrm{SD}, 6.58^{\circ}\right)$. Concerning FEMA, the average Cobb angle prior to bracing for the 15 patients was $31^{\circ}$ for the main thoracic (MT) curve and $32^{\circ}$ for the thoracolumbar/lumbar (TL/L) curve. The New Brace (FEMA) reduced Cobb angles by $42 \%$ (39\% for the MT curve and $49 \%$ for the TL/L curve), which were predicted with a difference inferior to $5^{\circ}$ by the simulation. The standard brace (Boston type) reduced these angles by $43 \%$ [11]. In-brace corrections differed widely when the different approaches were compared. In-brace corrections in the $\mathrm{CBA}$ was $66 \%$ of the initial value and in-brace correction in the FEMA was $42 \%$ of the initial value when not wearing the brace. Statistical comparison of the extent of in-brace corrections of CBA (sample derived from [2]) with FEMA (sample derived from [11]) revealed no significant differences ( $t=-1.46, p=0.05, z=1.96$ ).

\section{Discussion}

The CBA with respect to in-brace correction seems supe- rior to the FEMA (Figs. 5, 6). As in-brace correction and compliance determine the outcome of brace treatment [3], the CBA seems promising. The CBA was derived from the latest refinment of the Cheneau bracing, which reportedly has the best in-brace correction exceeding $50 \%$ of the initial value of $66 \%$ [2].

The differences of in-brace corrections between CBA and FEMA did not reach the level of significance. However, this may have reflected the limited number of patients. The two samples were comparable in Cobb angle $\left(31^{\circ}\right.$ in both).

CBA is readily available and can be used by submitting the patients' data, anthropometric measurements and pictures (including the X-ray); or by submitting a scan file of the patient together with the patients' data and pictures (including the X-ray). No stereo X-rays are necessary for CBA. Like FEMA, CBA can be improved by time to achieve a better in-brace correction.

Brace treatment can effectively halt progression. A recent randomized controlled trial [13] demontrated that $72 \%$ of the United States and Canadian population compliant with SRS inclusion criteria on bracing [14] did not progress using the standard thoracolumbosacral orthosis (TLSO, mainly Boston type). As FEMA does not seem to provide better in-brace corrections than the standard TLSO bracing in the United States [11], we would not be able to predict an improved outcome when comparing FEMA to the standard TLSO brace. 

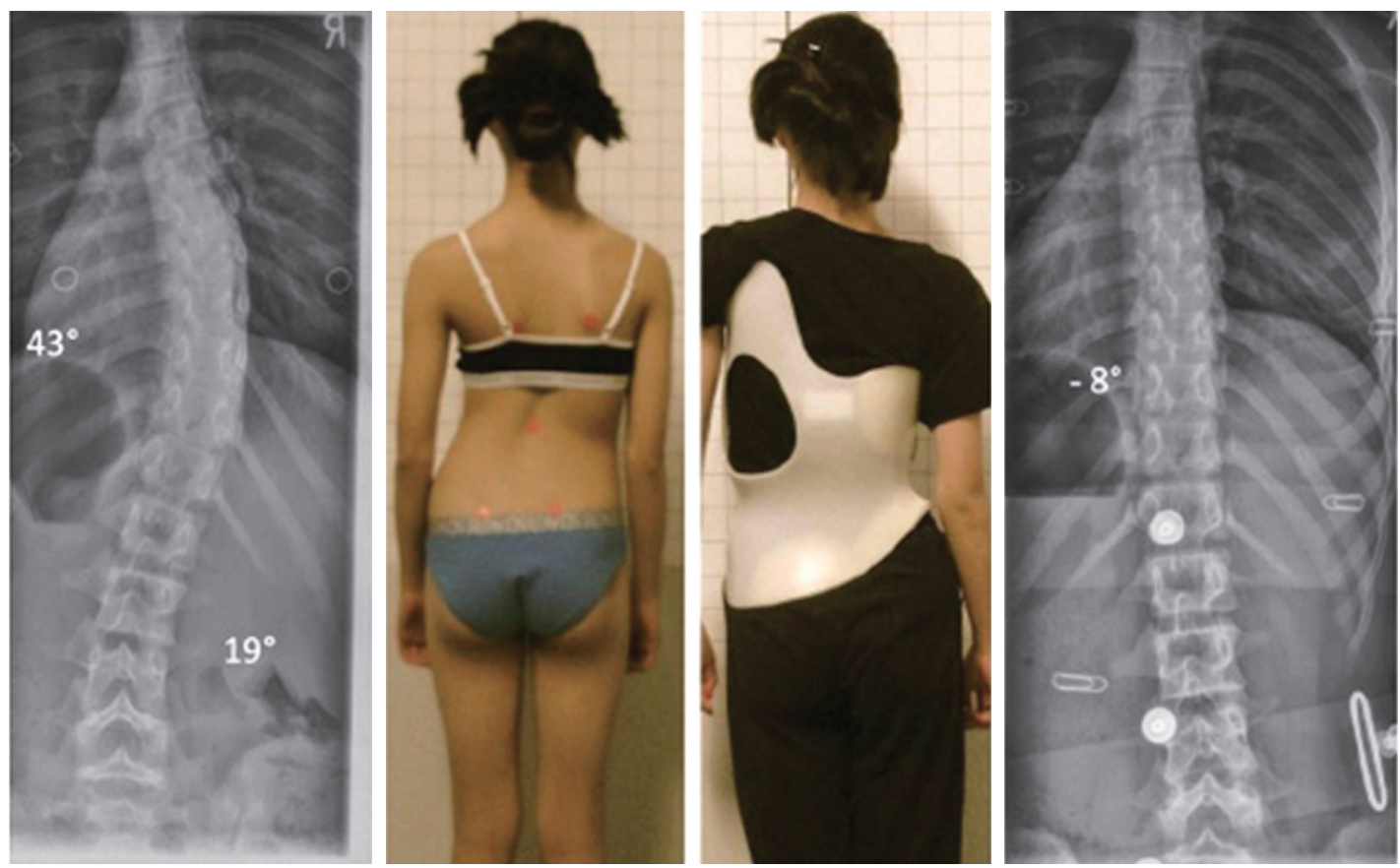

Fig. 5. Brace from a patient with a full correction of the single thoracic curve pattern. This patient, from New Zealand, was 12 years old with Tanner II-III and so was still rather flexible. Reprinted from Weiss et al. [2], according to the Creative Commons License of OA Publishing London.
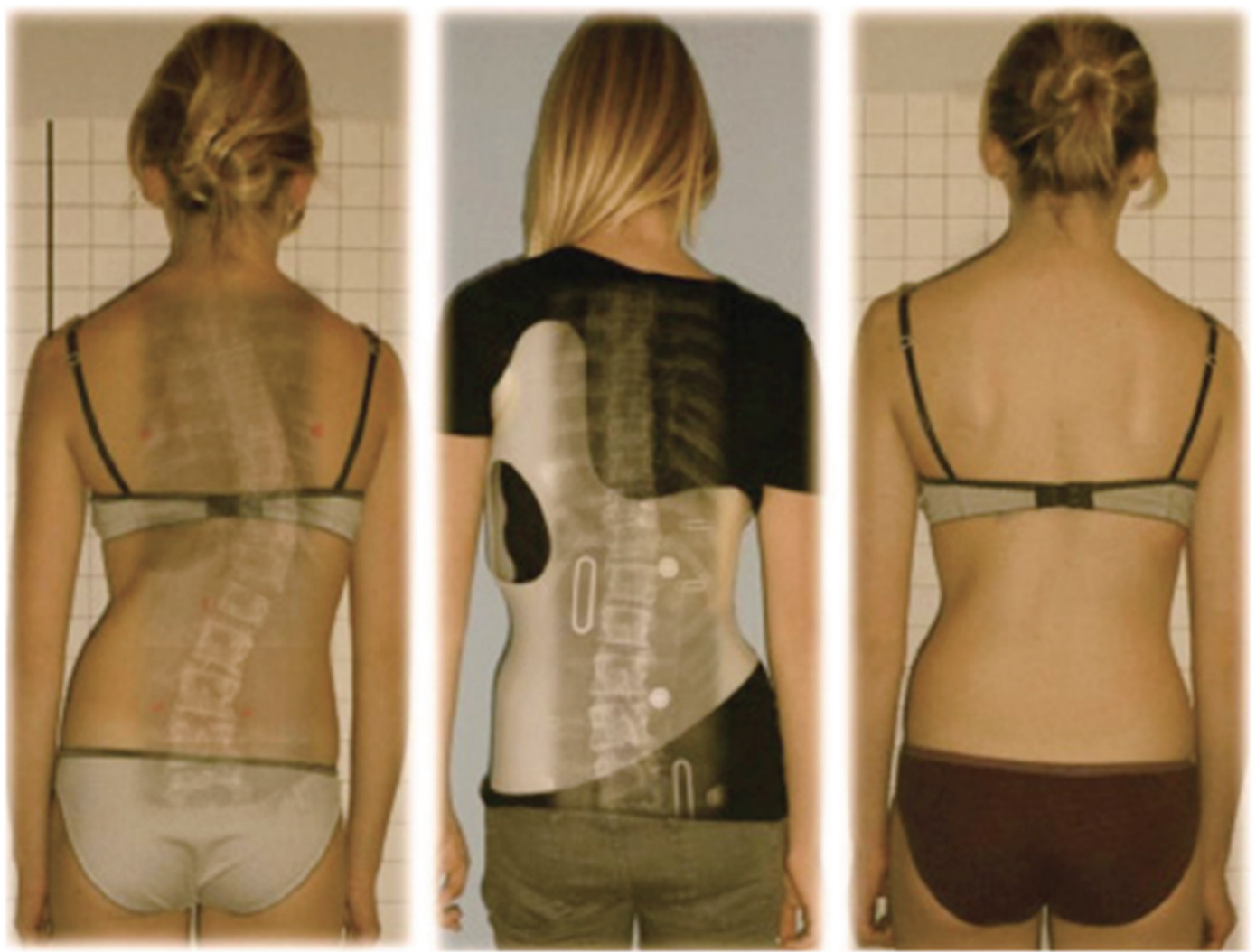

Fig. 6. Clinical result of a 15-year-old patient with a 42-degree Cobb angle at the start of treatment with marked progression within a few weeks. Right: clinical result following 6 months of treatment after outgrowing her first brace. Pelvic width has increased as compared to the photo at far left revealing skeletal immaturity at the start, age 15, although normally 15-year-old girls are nearly fully grown (to 99\%). Reprinted from Weiss et al. [2], according to the Creative Commons License of OA Publishing London. 
A retrospective series using CBA in a sample fulfilling the SRS inclusion criteria of the studies on bracing revealed a success rate exceeding 95\% $[14,15]$. This was confirmed in another study using the correction principles according to Cheneau [16]. Recently, it was reported that $33 \%$ of a population demonstrating improvements of Cobb angle exceeding 5 degrees after weaning off the CBA braces, with none of the subjects requiring surgery [17].

Considering that in-brace correction (and compliance) determine the end result of bracing in the treatment of scoliosis [3], we acknowledge that CBA is superior to FEMA and to the standard brace applications used in the United States and Canada [13]. For the best possible results in terms of avoiding surgery at this stage, CBA should be preferred [15].

FEMA is closely attached to the exterior surface of the patient. How often such a brace has to be renewed during growth is relevant an unanswered question. This also applies to braces manufactured using 3D printing [18]. Even a marginal gains in pelvic size will make a new brace necessary, because the FEMA and the actual 3D printed models fully wrap the body in the brace, leaving no room for growth (Fig. 7A).
The actual models of the CBA [12]-the Gensingen brace series-have the advantage of covering only one pelvic half, which preserves room for the corrective movement and an overlap of the ventral closure. Therefore, we can predict longer usage of the latter brace before outgrowth (Figs. 4, 7).

\section{Conclusions}

Considering that in-brace correction (and compliance) determine the end result of bracing in the treatment of scoliosis [3], currently the CBA approach of bracing scoliosis is superior to FEMA and the standard brace applications as used in the United States and Canada.

The actual models of the CBA-the Gensingen brace series-have the advantage of covering one pelvic half, which preserves room for the corrective movement and overlap of the ventral closure. Therefore, longer usage of the latter brace before outgrowth is likely.

\section{Conflict of Interest}

Hans-Rudolf Weiss is receiving financial support for attending symposia and receives royalties from Koob
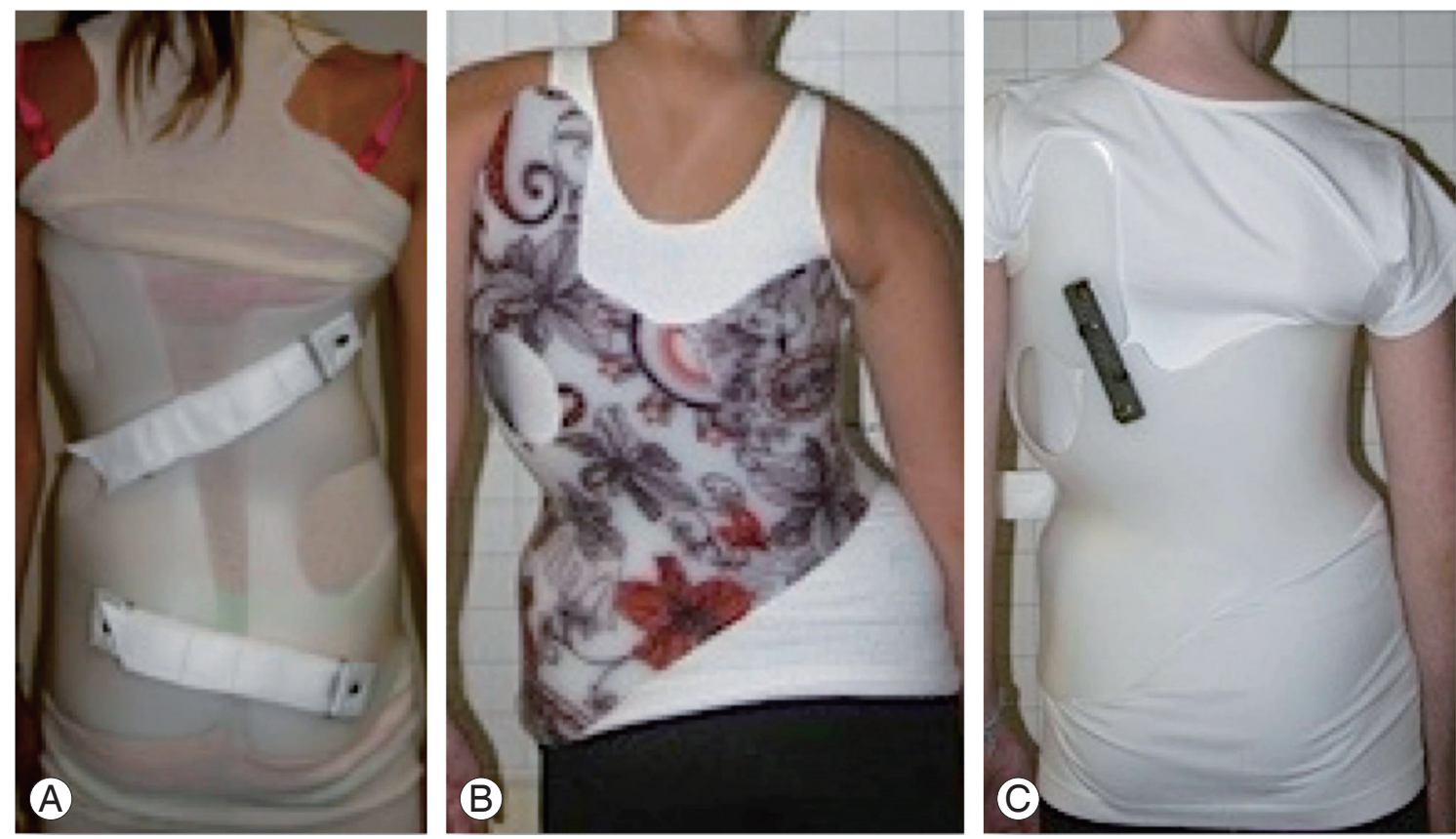

Fig. 7. (A) Finite element modelling approach brace with little asymmetry (reprinted from Clin et al. [11]). (B, C) The classification based approach braces according to the Gensingen brace computer aided design/computer aided manufacturing (CAD/CAM) library with clear asymmetry and good in-brace correction. 
GmbH \& Co KG.

\section{Acknowledgments}

All authors contributed to conception and design, manuscript preparation, read and approved the final manuscript. The authors are thankful to Dr. SY Ng, Hong Kong for his helpful advice and for helping with copyediting of this paper.

\section{References}

1. Weiss HR, Moramarco M. Scoliosis-treatment indications according to current evidence. OA Musculoskelet Med 2013;1:1.

2. Weiss HR, Seibel S, Moramarco M, Kleban A. Bracing scoliosis: the evolution to CAD/CAM for improved in-brace corrections. Hard Tissue 2013;2:43.

3. Landauer F, Wimmer C, Behensky H. Estimating the final outcome of brace treatment for idiopathic thoracic scoliosis at 6-month follow-up. Pediatr Rehabil 2003;6:201-7.

4. Clin J, Aubin CE, Labelle H. Virtual prototyping of a brace design for the correction of scoliotic deformities. Med Biol Eng Comput 2007;45:467-73.

5. Labelle H, Bellefleur C, Joncas J, Aubin CE, Cheriet F. Preliminary evaluation of a computer-assisted tool for the design and adjustment of braces in idiopathic scoliosis: a prospective and randomized study. Spine (Phila Pa 1976) 2007;32:835-43.

6. Clin J, Aubin CE, Parent S, Sangole A, Labelle H. Comparison of the biomechanical 3D efficiency of different brace designs for the treatment of scoliosis using a finite element model. Eur Spine J 2010;19: 1169-78.

7. Clin J, Aubin CE, Parent S, Labelle H. Biomechanical modeling of brace treatment of scoliosis: effects of gravitational loads. Med Biol Eng Comput 2011;49: 743-53.

8. Clin J, Aubin CE, Sangole A, Labelle H, Parent S. Correlation between immediate in-brace correction and biomechanical effectiveness of brace treatment in adolescent idiopathic scoliosis. Spine (Phila Pa 1976)
2010;35:1706-13.

9. Desbiens-Blais F, Clin J, Parent S, Labelle H, Aubin CE. New brace design combining CAD/CAM and biomechanical simulation for the treatment of adolescent idiopathic scoliosis. Clin Biomech (Bristol, Avon) 2012;27:999-1005.

10. Weiss HR, Rigo M, Cheneau J. Praxis der CheneauKorsettversorgung in der skoliosetherapie. 1st ed. Stuttgart: Thieme; 2000.

11. Clin J, Cobetto N, Desbiens-Blais F, et al. Advanced brace design combining CAD/CAM and biomechanical simulation for the treatment of adolescent idiopathic scoliosis. Proceeding of the OTWorld 2014; 2014 May 13; Leipzig, Germany.

12. Weiss HR. Standardising the treatment with Cheneau braces via CAD: pospects and risks. OA Musculoskelet Med 2014;2:10.

13. Weinstein SL, Dolan LA, Wright JG, Dobbs MB. Effects of bracing in adolescents with idiopathic scoliosis. N Engl J Med 2013;369:1512-21.

14. Richards BS, Bernstein RM, D'Amato CR, Thompson GH. Standardization of criteria for adolescent idiopathic scoliosis brace studies: SRS Committee on Bracing and Nonoperative Management. Spine (Phila Pa 1976) 2005;30:2068-75.

15. Weiss HR, Werkmann M. Rate of surgery in a sample of patients fulfilling the SRS inclusion criteria treated with a Cheneau brace of actual standard. Stud Health Technol Inform 2012;176:407-10.

16. De Giorgi S, Piazzolla A, Tafuri S, Borracci C, Martucci A, De Giorgi G. Cheneau brace for adolescent idiopathic scoliosis: long-term results. Can it prevent surgery? Eur Spine J 2013;22 Suppl 6:S815-22.

17. Weiss HR, Seibel S, Kleban A. Deformity-related stress in a sample of patients with adolescent idiopathic scoliosis after brace weaning: a cross-sectional investigation. OA Musculoskelet Med 2014;2:5.

18. Chavez E. A perfect fit: 3D systems unveils Bespoke 3D printed braces for chronic scoliosis [Internet]. New York: 3D Printing Industry; 2014 Jun 12 [cited 2015 May 29]. Available from: http://3dprintingindustry. com/2014/06/12/perfect-fit-3d-systems-unveilsbespoke-3d-printed-braces-chronic-scoliosis/. 